\title{
Exogenous capture accounts for fundamental differences between prosaccade and antisaccade performance
}

\author{
Allison T Goldstein, Terrence R Stanford, Emilio Salinas* \\ Department of Neurobiology and Anatomy, Wake Forest School of Medicine, 1 Medical Center \\ Blvd., Winston-Salem, NC 27157-1010, USA
}

${ }^{*}$ Correspondence should be addressed to:

Emilio Salinas

Department of Neurobiology and Anatomy

Wake Forest School of Medicine

1 Medical Center Blvd.

Winston-Salem, NC 27157-1010, USA

(336) 713-5176

esalinas@wakehealth.edu

www.urgentchoicelab.org

\begin{abstract}
Abbreviations: CAS, compelled antisaccade; CPS, compelled prosaccade; CI, confidence interval; ERI, exogenous response interval; FEF, frontal eye field; rPT, raw processing time; RT, reaction time; SC, superior colliculus; SD, standard deviation; SE, standard error
\end{abstract}

Running title: Exogenous contributions to prosaccades versus antisaccades

Acknowledgments: We thank Denise Anderson for technical and logistical assistance.

Grants: Research was supported by the NIH through grant R21MH120784 from the NIMH, grant R01EY025172 from the NEI, and training grant T32NS073553-01 from the NINDS.

Disclosures: The authors declare no conflicts of interest, financial or otherwise.

Author contributions: ES and TRS designed the research; AG collected data; AG and ES analyzed data; AG, ES and TRS wrote the manuscript.

Keywords: attention, capture, decision making, inhibitory control, mental chronometry, salience 
Oculomotor circuits generate eye movements based on the physical salience of objects and current behavioral goals, exogenous and endogenous influences, respectively. However, the interactions between exogenous and endogenous mechanisms and their dynamic contributions to target selection have been difficult to resolve because they evolve extremely rapidly. In a recent study (Salinas et al., 2019), we achieved the necessary temporal precision using an urgent variant of the antisaccade task wherein motor plans are initiated early and choice accuracy depends sharply on when exactly the visual cue information becomes available. Empirical and modeling results indicated that the exogenous signal arrives $\sim 80 \mathrm{~ms}$ after cue onset and rapidly accelerates the (incorrect) plan toward the cue, whereas the informed endogenous signal arrives $\sim 25$ ms later to favor the (correct) plan away from the cue. Here, we scrutinize a key mechanistic hypothesis about this dynamic, that the exogenous and endogenous signals act at different times and independently of each other. We test quantitative model predictions by comparing the performance of human participants instructed to look toward a visual cue versus away from it under high urgency. We find that, indeed, the exogenous response is largely impervious to task instructions; it simply flips its sign relative to the correct choice, and this largely explains the drastic differences in psychometric performance between the two tasks. Thus, saccadic choices are strongly dictated by the alignment between salience and behavioral goals.

The oculomotor system of primates chooses a new target to look at every 200-250 ms. Two components of visuospatial attention contribute to this selection process: exogenous mechanisms, which respond to the physical salience of the objects in view, and endogenous mechanisms, which prioritize those objects according to their relevance to current behavioral goals (Itti and Koch, 2001; Theeuwes, 2010; Wolfe and Horowitz, 2017). Although the qualitative distinctions between these mechanisms are well established, resolving their dynamic interactions and specific, real-time contributions to the selection process has been difficult because that requires temporal resolution far greater than can be achieved with traditional psychophysical tasks.

In a recent study (Salinas et al., 2019), we used an urgent version of the antisaccade task (Fig. 1a) to dissociate the exogenous and endogenous contributions to performance with millisecond precision. In the urgent task design (Stanford and Salinas, 2021), the go signal that commands the subject to respond is given before the visual cue that specifies the correct choice (an eye movement away from the cue, in this case). This way, motor plans are initiated early, so participants guess on some trials and make informed choices on others, but critically, each outcome depends fundamentally on how much time the participant has to see the cue before initiating a response, a quantity that we call the raw processing time (rPT). If choice accuracy is then plotted as a function of rPT, the result is a novel psychometric measure - the tachometric curve - that describes the participant's perceptual performance on a moment-by-moment basis (Stanford and Salinas, 2021).

In the urgent antisaccade experiment (Salinas et al., 2019), the tachometric curve revealed a unique feature that can be described as an 'attentional vortex,' a range of processing times roughly between 90-130 ms in which performance plummetted to nearly $0 \%$ correct (Fig. $2 \mathrm{~d}$ ). In other words, there was a range of conditions (i.e., states of motor preparation) indexed by processing time over which the cue onset would almost inevitably capture the saccade and lead to an error. Thereafter, for $\mathrm{rPT} \gtrsim 140 \mathrm{~ms}$, accuracy increased steadily and reached nearly $100 \%$ correct.

We interpreted these data as the result of a conflict between the early attentional pull toward the cue, which is salience-driven, involuntary, and transient, and the later intention to look away, which is task-dependent, voluntary, and sustained. This conflict is hardly detectable during active fixation, but becomes obvious when saccade plans are ongoing. As such, it was instantiated within a race-to-threshold model in which two opposing motor plans compete to trigger an eye movement to the right or to the left $(\mathrm{Fig} 2 \mathrm{a}-\mathrm{c})$. As elaborated below, these plans initially advance 

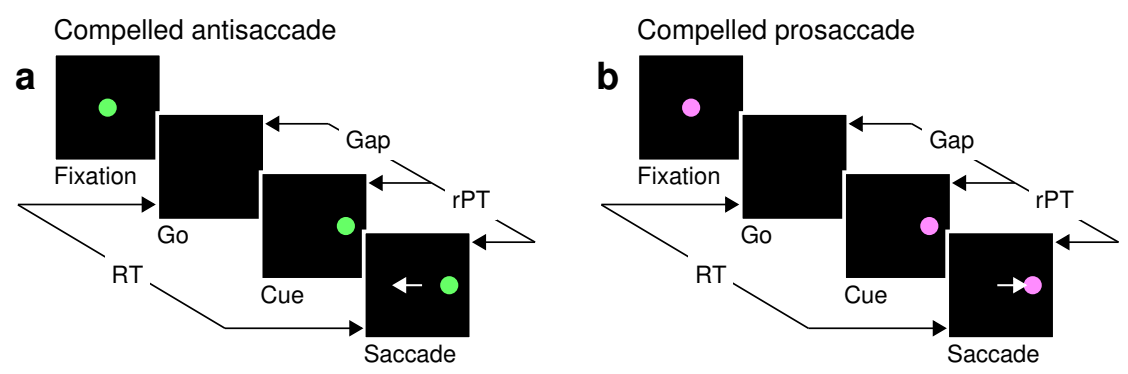

Figure 1. The urgent tasks. a, The compelled antisaccade (CAS) task. After a fixation period (500, 600, or $700 \mathrm{~ms}$ ), the central fixation point disappears (Go), instructing the participant to look to the left or to the right within $425 \mathrm{~ms}$. The cue is revealed later $\left(\mathrm{Cue}, \pm 8^{\circ}\right)$, after a time gap that varies unpredictably across trials (Gap, 0-350 ms). The correct response is an eye movement away from the cue, to the diametrically opposite location (Saccade, white arrow). $\mathbf{b}$, The compelled prosaccade (CPS) task. The sequence of events is the same as for the compelled antisaccade task, except that the correct response is an eye movement toward the cue. In all trials, the cue location and gap are selected randomly; the reaction time (RT) is measured between the onset of the go signal and the onset of the saccade; and the raw processing time $(\mathrm{rPT})$ is measured between cue onset and saccade onset (calculated as RT - gap).

at randomly-drawn rates, but afterward, time permitting, they are steered by perceptual information. The exogenous signal arrives early ( $\sim 80 \mathrm{~ms}$ after cue onset) and rapidly accelerates the plan toward the cue regardless of task instructions; the endogenous signal arrives ( $\sim 25 \mathrm{~ms})$ later to accelerate the correct plan and decelerate the incorrect one as stipulated by the task. These dynamics replicated all aspects of the behavioral data in great detail.

Here, we leverage this quantitative framework to further test a fundamental conclusion derived from that study: that the exogenous modulation of ongoing motor plans is highly stereotyped and largely independent of behavioral context. We use the antisaccade model to predict the performance that should be observed during an urgent prosaccade task (Fig. 1b) if this independence hypothesis is correct, and we test the parameter-free predictions in human participants performing both urgent prosaccades and urgent antisaccades. In essence, the exogenous modulation should evolve with the same timecourse regardless of task instructions, and should favor the motor plan toward the cue in the same way whatever the state of development of the competing motor plans.

To anticipate the main findings, the differences between pro- and antisaccade performance are dramatic, but can be largely accounted for by a fixed exogenous response that is either aligned or misaligned with the endogenously defined goal.

\section{Results}

\section{A quantitative framework for urgent antisaccade and prosaccade performance}

In our previous study (Salinas et al., 2019), we developed a neurophysiologically feasible model that quantitatively replicated the rich psychophysical data set obtained in the compelled antisaccade task (the CAS model). Now, with a minimal modification, we generate a model of the compelled prosaccade task (the CPS model). The modification corresponds to simply making the top-down, endogenous response favor a saccade to the cue instead of away from the cue without changing anything else.

The CAS model consists of two variables, $r_{L}$ and $r_{R}$, that represent oculomotor activity favoring saccades toward left and right locations (Fig. 2a-c, red and black traces). The first one of these motor plans to exceed a fixed threshold level (Fig. 2a-c, dashed lines) triggers a saccade, to the left 

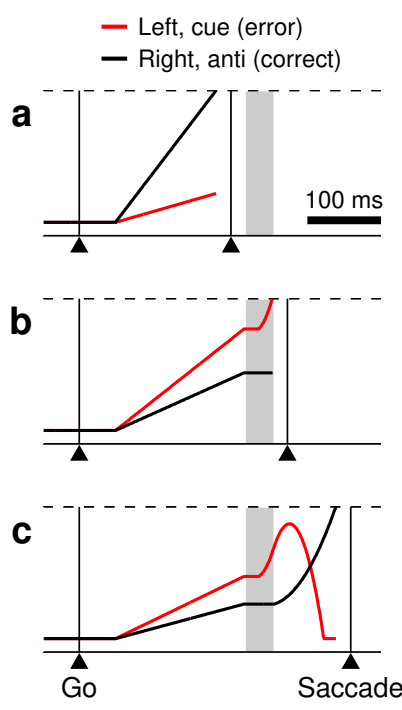
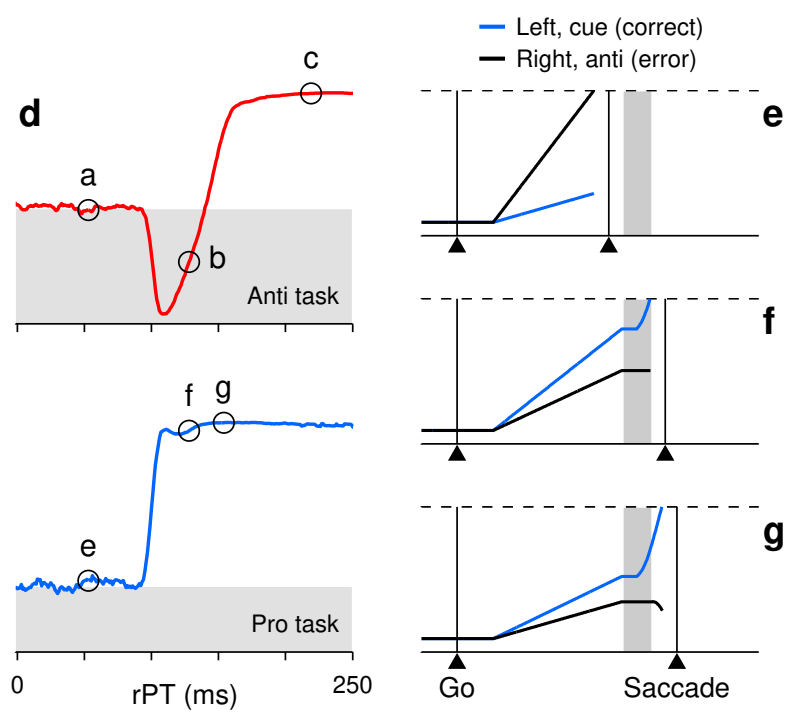

Figure 2. Turning a model of antisaccade performance into one of prosaccade performance. a-c, Three single antisaccade trials simulated with the CAS model. The cue is assumed to be on the left and the gap is $150 \mathrm{~ms}$. Traces show motor plans $r_{L}$ toward the left (red, incorrect) and $r_{R}$ toward the right (black, correct) as functions of time. During the exogenous response interval (ERI, gray shade), the plan toward the cue accelerates. After the ERI, the incorrect plan decelerates and the correct one accelerates. A saccade is triggered a short efferent delay after activity reaches threshold (dashed lines). Examples include a correct, short-rPT guess ( $\mathbf{a}, \mathrm{rPT}=56 \mathrm{~ms})$; an incorrect, captured saccade $(\mathbf{b}, \mathrm{rPT}=133 \mathrm{~ms})$; and a correct, informed choice (c, rPT = $219 \mathrm{~ms}$ ). d, Simulated tachometric curves for the CAS (top, red) and CPS tasks (bottom, blue). The $\mathrm{x}$ and $\mathrm{y}$ axes correspond to raw processing time and fraction of correct choices, respectively. Gray shades indicate below-chance performance. $\mathbf{e}-\mathbf{g}$, Three single prosaccade trials with the same initial motor plans as in a-c but simulated with the CPS model. They include an incorrect, short-rPT guess (e, rPT $=56 \mathrm{~ms})$; a correct, captured saccade $(\mathrm{f}, \mathrm{rPT}=133 \mathrm{~ms})$; and a correct, informed choice $(\mathrm{g}$, $\mathrm{rPT}=149 \mathrm{~ms})$. The pro and anti simulations differed only in the movement that was considered correct, which amounted to swapping the motor plans that were endogenously accelerated and decelerated.

if $r_{L}$ crosses the threshold first, or to the right if $r_{R}$ does. In each trial, $r_{L}$ and $r_{R}$ start increasing in response to the go signal, initially advancing with randomly-drawn buildup rates. This ramping process may end in a random choice (i.e., a guess; Fig. 2a) if the gap interval is long and/or one of the initial buildup rates is high enough. Otherwise, time permitting, the arrival of the cue information modifies the ongoing motor plans in two ways.

First, when the cue onset is detected by the oculomotor circuit, the motor competition is biased toward the cue location during a time period that we refer to as the exogenous response interval, or ERI (Fig. 2a-c, gray shades). Specifically, this means that, during the ERI, the motor plan toward the cue is first briefly halted and then accelerated (Fig. 2b, c, red traces during gray interval), whereas the motor plan toward the opposite, or 'anti,' location is halted throughout (Fig. 2b, c, black traces during gray interval). These modulations of the ongoing motor activity constitute the involuntary, exogenous response.

Then, after the ERI ends, once the cue location has been interpreted in accordance to task rules as "opposite to the target," the motor selection process is steered toward the correct choice. Specifically, the (erroneous) plan toward the cue is decelerated (Fig. 2c, red trace after gray interval) and the (correct) plan away from the cue is accelerated (Fig. 2c, black trace after gray interval). These modulations informing the correct choice constitute the top-down, endogenous response.

With these elements in place, the CAS model was able to reproduce the full range of psy- 
chophysical results obtained in the urgent antisaccade task, both on average and in individual participants (with parameter values adjusted accordingly; Salinas et al., 2019). In particular, the model simulations accurately replicated the tachometric curve (Fig. 2d, top), i.e., the function relating choice accuracy to rPT, which is the key behavioral metric in such urgent tasks. In the rest of the paper we consider the CAS model with parameters fitted to the pooled data from 6 participants (Salinas et al., 2019, Table 1, high-luminance cue). The tachometric curve simulated for this case (Fig. 2d, top) directly illustrates the conflicting interaction between exogenous and endogenous mechanisms that characterizes the CAS task: When the cue is seen for less than $90 \mathrm{~ms}$ or so (as in Fig. 2a), the saccades are uninformed and the success rate stays near chance. When the cue is seen for more than $140 \mathrm{~ms}$ or so (as in Fig. 2c), most saccades are informed and the success rate rises steadily above chance and toward $100 \%$ correct. But in between, performance drops to nearly $0 \%$ correct, as a large fraction of saccades are drawn to the wrong location. Those saccades are captured by the cue (as in Fig. $2 b$ ).

To generate predictions about performance in prosaccade trials, we modified the CAS model so that the endogenous signal accelerated the motor plan toward the cue and decelerated the competing plan toward the anti location. That is, we simply swapped the target and distracter designations for the two locations without altering any parameter values. In this way, in the resulting CPS model the exogenous modulation during the ERI still boosts the motor plan toward the cue, just as it did originally (Fig. 2f, compare to panel b). Crucially, though, those motor plans that are propelled past the threshold, which previously resulted in erroneous captured saccades, now correspond to correct choices. Furthermore, the informed endogenous signal now typically acts to reinforce the motor plan that is already ahead by the end of the ERI (Fig. $2 g$, compare to panel c). All this results in a predicted tachometric curve for prosaccade performance for which the success rate grows steadily with rPT, rising very early and very steeply (Fig. $2 \mathrm{~d}$, bottom).

\section{Specific model predictions}

The fundamental assumption behind the CPS model just described is that the exogenous response is entirely insensitive to behavioral context, so the endogenous signal reinforces the correct choice in exactly the same way in the pro and anti tasks - i.e., the acceleration and deceleration terms simply swap their spatial assignments - regardless of the state of development of the motor alternatives. This is one extreme in the spectrum of possible outcomes; opposite to this 'fullindependence' scenario is the possibility that the exogenous and endogenous modulations are much different in one task than in the other; and, of course, one or both of these mechanisms could also change modestly across conditions. Hence, the more general question is, to what degree are the exogenous and endogenous mechanisms sensitive to behavioral context? We use the CPS model as a benchmark because it instantiates a conceptually simple hypothesis (independence) to yield parameter-free, quantitative predictions - which, as it turns out, to a first approximation are fairly accurate.

The CAS and CPS models make two specific, clear-cut predictions. The first one is that the upswing in performance during prosaccade trials should follow the same timecourse as the initial downswing in performance during antisaccade trials (Fig. 3a, b). In other words, the initial departure from chance should exhibit the same dependence on rPT but going in opposite directions, toward $100 \%$ versus toward $0 \%$ correct. This is a direct consequence of the exogenous response reinforcing the motor plan toward the cue with the same timing and intensity in the two tasks.

The second prediction is about the rise toward asymptotic performance when there is a motor bias, that is, when the participant tends to guess more toward one side than the other. If such a bias exists, then during antisaccade trials in which the cue happens to appear on the preferred 

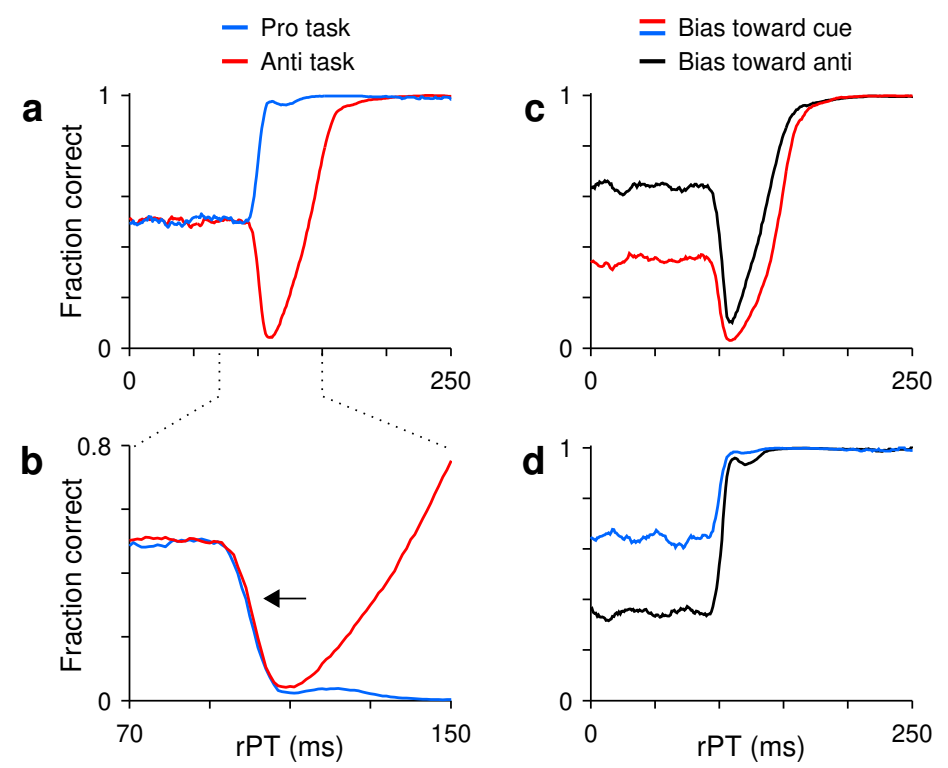

Figure 3. Model predictions. a, Simulated tachometric curves for the pro- (blue trace) and antisaccade tasks (red trace). Same curves as in Fig. 2d, but superimposed. b, Same data as in a, but shown over a smaller rPT range and with the prosaccade curve inverted (blue trace) relative to the chance level. Note that the initial departure from chance follows the same timecourse for the two tasks (arrow). c, Antisaccade tachometric curves conditioned on cue location. Traces are expected results if the participant consistently guesses in one direction and trials are split into two groups: with the cue on the preferred side (red trace) or with the cue on the non-preferred side (black trace). Note that the rise toward asymptotic performance occurs later when the initial motor bias is in the direction of the cue. $\mathbf{d}$, As in c, but for the prosaccade task. Note that, in this case, the rise in performance is similar regardless of the initial bias.

guessing side, the rise in success rate is expected to occur late (Fig. 3c, red trace). In contrast, during antisaccade trials in which the cue happens to appear opposite to the preferred guessing side, the success rate should rise earlier (Fig. 3c, black trace). This difference arises because the exogenous and endogenous modulations are always opposite, so it is more difficult for the endogenous signal to prevent a capture when the exogenous response and the motor bias reinforce each other. On the other hand, during prosaccade trials the rise in success rate is expected to be very similar regardless of where cue appears relative to the guessing direction (Fig. 3d). In this case, the exogenous and endogenous modulations are aligned and can easily overcome any bias in the initial motor plans, so the rise in success rate is always very steep.

These two predictions are complementary in that they probe bottom-up and top-down components of the selection process. The first one tests whether the sensory-driven exogenous response itself changes across tasks, whereas the second one tests whether the endogenously-driven recovery depends on the state of development of the competing motor alternatives.

\section{The exogenous response is highly insensitive to behavioral context}

To test the model predictions, 10 participants ( 6 female, 4 male) were recruited. The experimental procedures were largely the same as in the earlier report (Salinas et al., 2019) and are summarized in the Methods section. Any differences in approach are described there, along with analysis methods specific to this study.

All the participants performed pro and anti trials in two ways: in blocks of same-task trials, and in blocks of randomly interleaved pro- and antisaccades (Methods). The experiment was 

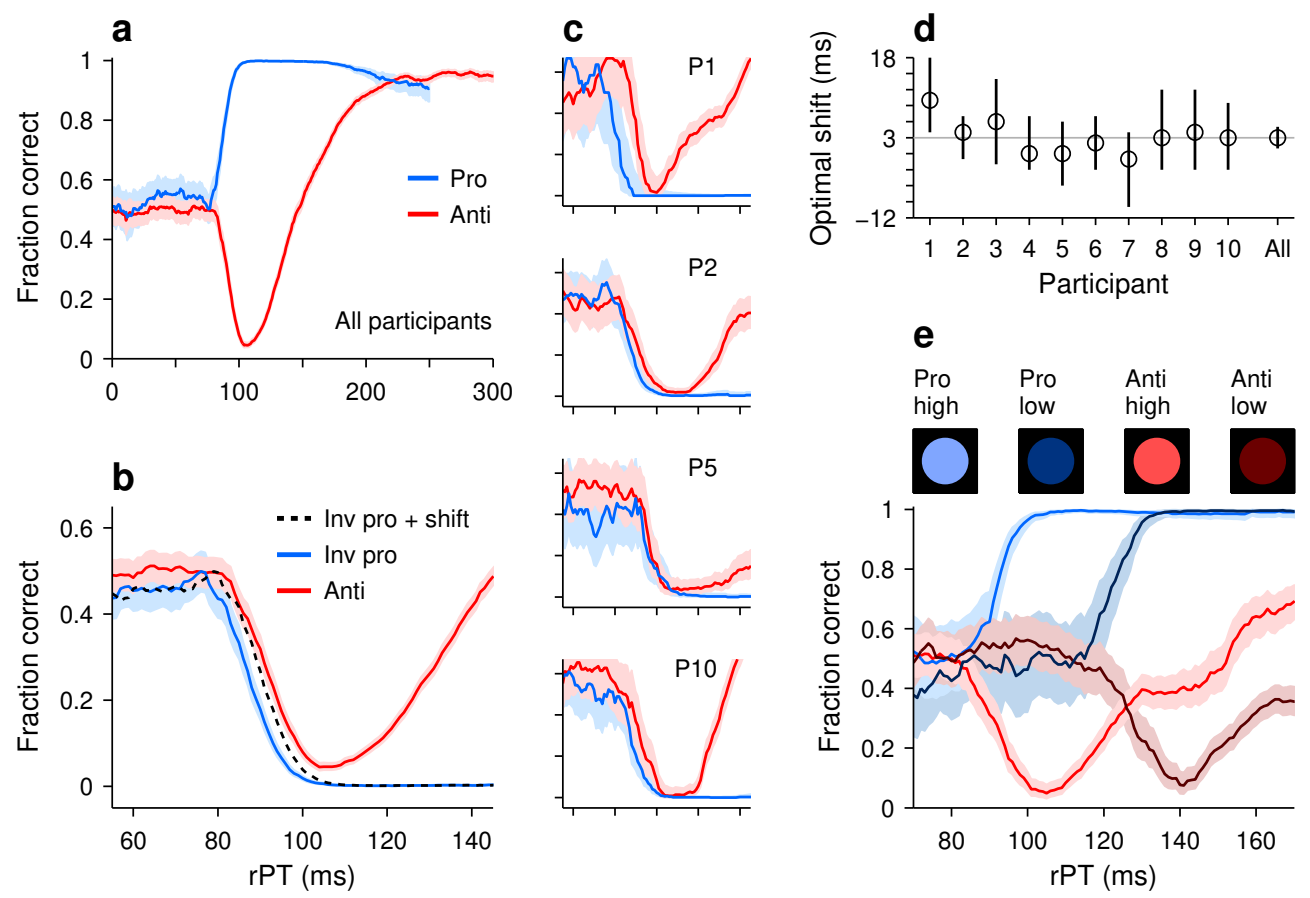

Figure 4. Early departure from chance during pro- and antisaccade performance. a, Tachometric curves for pro- (blue trace, 24,638 trials) and antisaccade trials (red trace, 22,608 trials) combined over 10 participants. Shades indicate $95 \%$ confidence intervals (CIs) across trials. b, Same data as in a, but shown over a smaller rPT range and with the prosaccade curve inverted (blue trace) relative to the chance level. The dotted line is the inverted prosaccade curve shifted by $3 \mathrm{~ms}$ along the $\mathrm{x}$ axis. c, Pro- and antisaccade curves for four individual participants. Same $\mathrm{x}$ and $\mathrm{y}$ axes as in $\mathbf{b}$, and same format. $\mathbf{d}$, Optimal shift for each participant. The optimal shift (along the rPT axis) minimized the difference between the inverted pro curve and the anti curve for rPTs in the $[55,105] \mathrm{ms}$ interval. The gray line marks the optimal shift $(3 \mathrm{~ms})$ for the pooled data in b. Error bars are 95\% CIs. e, Tachometric curves pooled from three participants tested with both the standard high-luminance cues (bright colors) and low-luminance cues (dark colors). Note that the luminance-driven right shift is similar for the pro and anti curves.

designed this way because it seemed possible that the two conditions could lead to major differences in performance, via task-switching costs, for instance (Wylie and Allport, 2000; Monsell, 2003). However, the observed differences were, in fact, very small (Fig. S2) and had no bearing on any of the subsequent analyses. Thus, in what follows, we consider all the trials of each task aggregated across blocked and interleaved conditions.

For the compelled antisaccade task, the pooled tachometric curve (Fig. 4a, red trace), generated by combining the data across participants, replicated our earlier result (Salinas et al., 2019). It exhibited the characteristic drop in success rate due to potent oculomotor capture in the range between 90 and $140 \mathrm{~ms}$, approximately, with the subsequent recovery reaching nearly $100 \%$ correct after $\sim 200 \mathrm{~ms}$ of cue viewing time. More importantly, for the compelled prosaccade task the shape of the pooled tachometric curve (Fig. 4a, blue trace) was as predicted by the model. In this case, the success rate rose steadily, early, and very steeply.

To evaluate this first prediction quantitatively, we compared the downswing in antisaccade performance to the upswing in prosaccade performance (Fig. 4b). First, the pro curve was inverted so that it decreased monotonically, and then a shift analysis was carried out to determine the optimal shift along the rPT axis that would maximize the overlap between the two curves 
(Methods). Whereas according to the prediction the optimal time shift should be zero (Fig. 3b, arrow), experimentally, we found that the optimal shift was, on average, $3 \mathrm{~ms}$ within a 95\% confidence interval (CI) of $[1,5] \mathrm{ms}$ (Fig. 4b, d). That is, in prosaccade trials, the effect of the exogenous response manifested about $3 \mathrm{~ms}$ earlier than in antisaccade trials. This time difference was of comparable magnitude across individual participants (Fig. 4c), which had optimal shifts in the [-1, 10] ms range (Fig. 4d). Also, the result was very similar when green and pink cues were assigned to pro and anti trials, respectively (participants 1-5; Methods), and when the color assignments were flipped (participants 6-10). In general, the upswing in prosaccade performance had just a slightly earlier onset than the downswing in antisaccade performance.

To confirm these results, we ran a variant of the experiment in which the cue could be of high or low luminance (Methods). We knew that, in the antisaccade task, less luminant cues produce capture at longer processing times (Salinas et al., 2019), so we reasoned that the rise in prosaccade performance should demonstrate the same temporal dependence - if it is driven by the same exogenous signal. The low luminance level was chosen to elicit a robust rightward shift of the anti tachometric curve (Salinas et al., 2019), and the high luminance level was identical to that in the main experiment. In this case, the cue luminance (high or low) and task type (pro or anti) were randomly and independently chosen in each trial, and data were collected from 3 participants (Methods).

As expected, in antisaccade trials the decrease in cue luminance resulted in a rightward shift of the tachometric curve (Fig. 4e, red curves), which was of $35 \mathrm{~ms}([33,38] \mathrm{ms} 95 \% \mathrm{CI})$, as determined by an optimal-shift analysis. And critically, a comparable delay was observed in prosaccade trials (Fig. 4e, blue curves), for which the lower luminance resulted in a shift of $30 \mathrm{~ms}([26,34] \mathrm{ms}$ $95 \% \mathrm{CI})$. The results were qualitatively the same for the three participants. In conclusion, the data indicate that, although they are not quite identical, the exogenous signal that rapidly propels prosaccade performance above chance must be very similar to the one that drives antisaccade performance below chance.

\section{Visuomotor dynamics revealed by motor biases}

The second prediction derived from the model applies when participants exhibit a motor bias, that is, a preference for looking to one side more than the other. Fortuitously, we did not have to manipulate the experimental conditions to induce such a preference because participants adopted one spontaneously.

When we analyzed our participants' performance as a function of their prior choices, we noticed a trend: their guesses in a given trial were generally toward the location where they should have responded in the previous trial (i.e., the prior target location). The psychometric manifestation of this tendency is a systematic deviation from chance observed when the trials are sorted according to the history of prior target locations. Specifically, the tachometric curve conditioned on a repeated target location (Fig. 5a, green trace) demonstrated a positive offset (above chance) in the range of processing times for which participants must guess (rPT $\lesssim 80 \mathrm{~ms}$ ), whereas the tachometric curve conditioned on a switch in target location (Fig. 5a, magenta trace) demonstrated a negative offset (below chance) in the same range. This effect was cumulative; the more target repetitions, the larger the offsets (Fig. 5, compare across columns). It was also observed in most participants (Fig. S3), and importantly, in both pro and anti trials.

Given this motor bias, we analyzed the tachometric curves conditioned on prior target location and measured the rise point in each case; for anti trials, this is the rPT at which the success rate is halfway between minimum and asymptotic (Fig. 5a-c, gray vertical shades), and for pro trials, it is the rPT at which the success rate is halfway between chance and asymptotic (Methods). We 

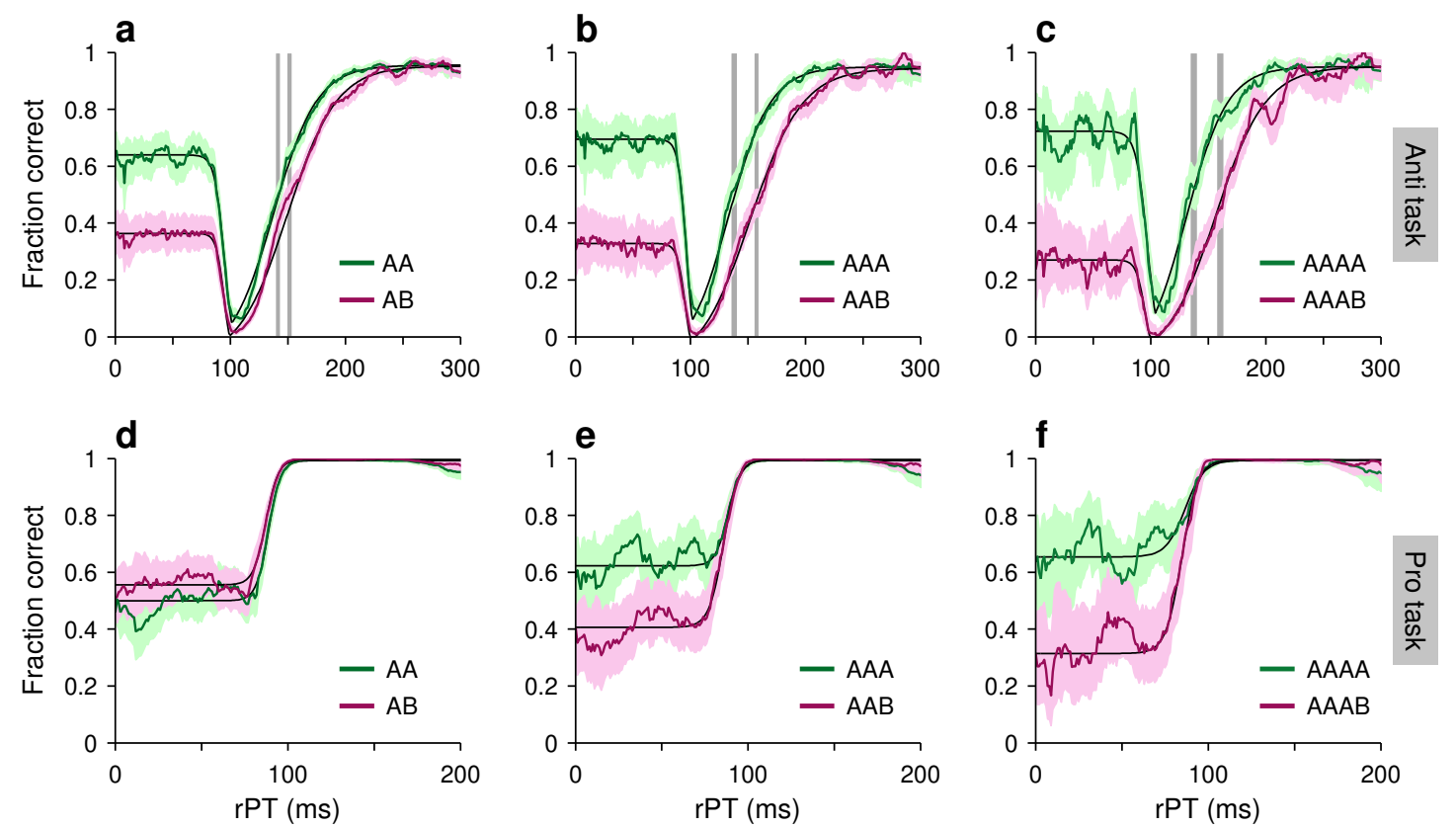

Figure 5. Tachometric curves conditioned on target-location history. Each panel shows choice accuracy as a function of processing time when the target location in a given trial was the same as in previous trials (green curves) or when it was different (magenta curves). Note that the participants' guesses (rPT $\lesssim 80 \mathrm{~ms}$ ) tended to be toward the previous target location. a-c, Performance in the compelled antisaccade task conditioned on the history of prior target locations going back 1 (a), 2 (b), or 3 trials (c). A and B labels stand for left or right target locations, and indicate patterns of repeats (AA, AAA, AAAA) or switches in location (AB, $\mathrm{AAB}, \mathrm{AAAB}$ ) preceding each choice. Continuous black curves are fits to the empirical data. Gray vertical shades indicate $95 \%$ CIs for the curve rise points. $\mathbf{d}-\mathbf{f}$, As in $\mathbf{a}-\mathbf{c}$, but for the compelled prosaccade task. Results are for data pooled across participants.

found that both aspects of the second model prediction were correct. In the antisaccade task, the rise toward asymptotic performance occurred late when the motor bias was aligned with the cue location (Fig. 5a-c, pink curves) and early when it was aligned with the anti, or target location (Fig. 5a-c, green curves); whereas in the prosaccade task, the rise was essentially the same in the two cases (Fig. 5d-f). It is notable that the effect in anti trials grew stronger as the bias became more extreme, and it is instructive to calculate by how much.

Consider the tachometric curves conditioned on one previous trial (Fig. 5a). As the chance level went from 0.36 ([0.34, 0.39] 95\% CI, AB trials) to 0.64 ([0.61, 0.66], AA trials), the rise point of the tachometric curve went from $154 \mathrm{~ms}([149,154] \mathrm{CI}, \mathrm{AB}$ trials) to $143 \mathrm{~ms}([139,144]$, AA trials). Taking the ratio of the differences gives a drop of approximately $39 \mathrm{~ms}$ when traversing the full chance range from 0 to 1 ; or equivalently, if the chance level increases by 0.1 , the rise point of the curve should drop by about $3.9 \mathrm{~ms}$. The reason why this number is of interest is that it can also be computed for the simulated data. To do this, we simply ran the model (as in Fig. 2c) with different amounts of motor bias, and for each run we tracked the chance level and the rise point of the simulated tachometric curve (Methods). According to this procedure, the predicted change in rise point over the full chance range was $40 \mathrm{~ms}([36,44]$ 95\% CI; Fig. S3b, blue line and circles), in excellent agreement with the experimental data. Although in this analysis there was considerable variance across individual participants (Fig. S3), the model prediction was very much in line with the data pooled across participants (Fig. S3b, compare triangles and blue circles). 

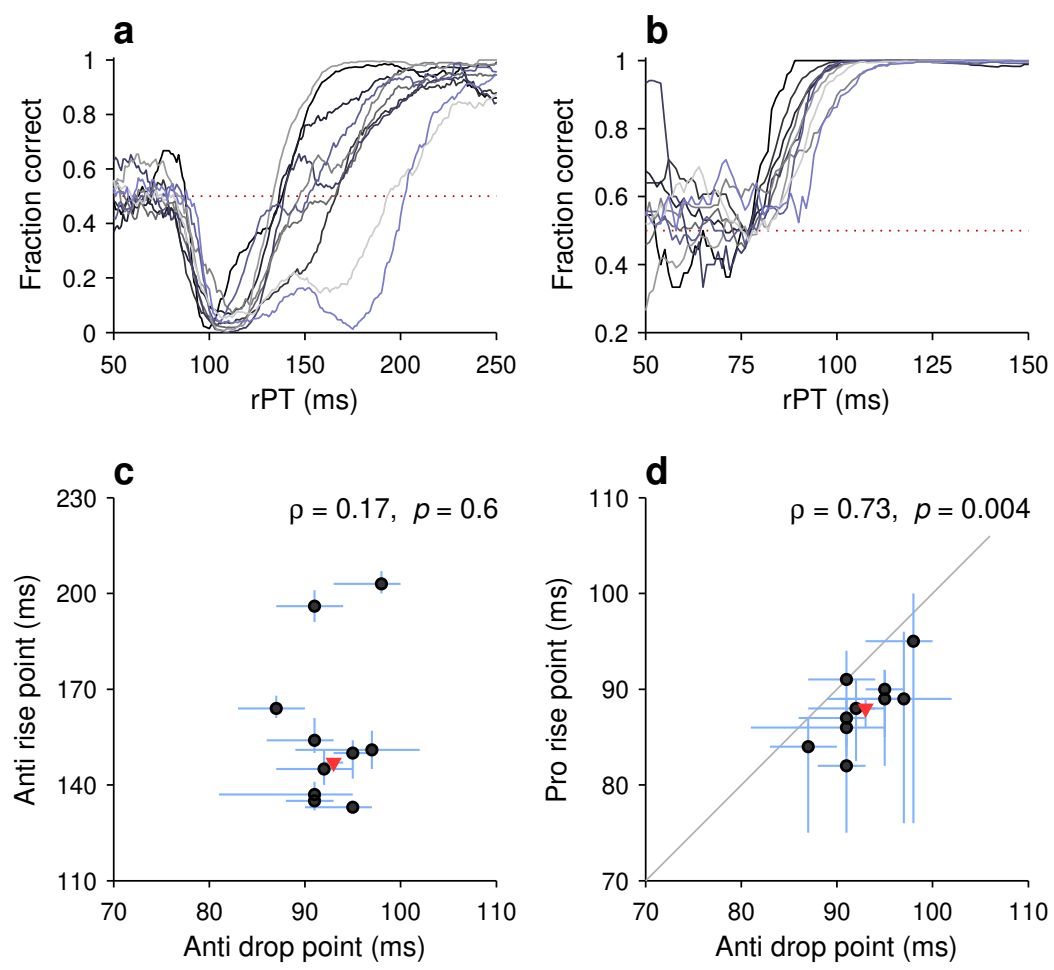

Figure 6. Variations in the timing of exogenous and endogenous modulation across participants. a, Tachometric curves in the compelled antisaccade task for all 10 participants. Dotted line indicates chance performance (0.5). $\mathbf{b}$, As in $\mathbf{a}$, but for the compelled prosaccade task. c, Comparison between drop point ( $\mathrm{x}$ axis; time of early, exogenously driven drop in performance) and rise point (y axis; time of later, endogenously driven rise in performance) in antisaccade trials. Each circle represents one participant. Lines indicate $95 \%$ CIs (from bootstrap). The Pearson correlation of the data $(\rho)$ and its significance (from permutation test) are indicated. The red triangle marks data from the pooled tachometric curve. d, Comparison between drop point in antisaccade trials ( $\mathrm{x}$ axis; time of early, exogenously driven drop in performance) and rise point in prosaccade trials (y axis; time of early rise in performance). Same format as in c. Note the positive correlation between the early responses in the two tasks.

\section{Consistency in the strength of exogenous capture across participants}

As expected, in the two tasks there were noticeable differences in performance between individual participants, and analysis of this variance provided further support for the two hypotheses underlying our model predictions.

For the antisaccade task, the tachometric curves of the participants were quite similar in their initial departure from chance, as saccades were increasingly captured by the cue, but they were much more variable during the later rise in success rate (Fig. 6a). Across the individual curves, the drop point (the rPT for which the success rate is halfway between chance and the minimum; Methods) ranged from 87 to $98 \mathrm{~ms}$, whereas the rise point ranged from 133 to $203 \mathrm{~ms}$. More importantly, the values for the drop point and the rise point were not significantly correlated (Fig. 6c; $p=0.6$, permutation test), consistent with the idea that, in each subject, the exogenous response that determines the former is independent of the endogenous process responsible for the latter.

For the prosaccade task, the rise point covered a range from 82 to $95 \mathrm{~ms}$ across participants (Fig. 6b), similar to that of the drop point in the anti task. More importantly, however, these two sets of values were significantly correlated (Fig. $6 \mathrm{~d} ; \rho=0.73, p=0.004$, permutation test). 
An alternate analysis based on optimal shifts instead of rise and drop points produced nearly identical correlation numbers. In summary, then, participants that showed an earlier departure from chance in one task also showed an earlier departure from chance in the other, consistent with the notion that, for each individual, the exogenous capture is fundamentally the same in the two tasks.

\section{Discussion}

We used the contrast between urgent pro- and antisaccade performance to test the idea that exogenous and endogenous contributions to saccadic target selection are largely independent. The cue onset and the endogenously defined goal were spatially congruent in one task but incongruent in the other, and yet, in both cases we observed a similarly steep increase in the frequency of saccades made toward the cue. As a result, there were striking differences in the proportion and timing of the correct responses across conditions (pro versus anti), but this was very much as predicted by our race-to-threshold model.

\section{Exogenous capture connects numerous oculomotor phenomena}

Given our modeling framework, the mechanistic intuition behind these results is straightforward: when saccade-related neural activity is ramping up, the cue onset can accelerate that activity past the threshold beyond which a saccade is inevitably produced - before top-down information can influence the choice. When the cue itself is not the target, as in the antisaccade task, this leads to so-called captured saccades, which are errors. But when the cue itself is the target, as in the prosaccade task, these same 'captured' saccades are correct. In either case, the eye movement is elicited very rapidly, after 75-100 ms of viewing time. The quantitative variations in this process across conditions were tiny, consistent with the hypothesis that the resulting bias on the motor selection process is associated with the detection of the cue and is largely independent of its behavioral relevance or meaning. Perhaps an appropriate descriptor for this effect, as it is thought to occur in oculomotor circuits, is 'exogenous capture.'

Understood this way - as an involuntary and stereotypical bias on motor planning that occurs when a stimulus is detected by oculomotor circuits - exogenous capture potentially encompasses a wide range of oculmotor phenomena (Salinas and Stanford, 2021). These include (1) oculomotor capture itself, which is observed when saccades are overtly directed toward salient distracters (Theeuwes et al., 1998, 1999); (2) express saccades, which are saccades triggered with extremely short latencies (Kalesnykas and Hallett, 1987; Paré and Munoz, 1996; Sparks et al., 2000); (3) exogenous attention, which improves perceptual performance at explicitly cued locations (Carrasco, 2011) and has neural correlates that are highly consistent with our framework (Busse et al., 2008; Herrington and Assad, 2010); and (4) attentional capture, which is typically observed when saccades to a target take longer in the presence of salient distracters (Ruz and Lupianñez, 2002; Theeuwes, 2010).

Exogenous capture is a potentially useful construct because it represents a specific hypothesis about the neural events that determine, or at least contribute significantly to, all of these behaviors. For example, the variations in RT that define attentional capture can be easily explained by our framework (Oor et al., 2021; Salinas and Stanford, 2021). In essence, when the distracter-driven acceleration of the cue-aligned plan is relatively weak (weaker than in Fig. 2c), most saccades are ultimately correct, but their onset is still delayed relative to a control condition with a less salient or absent distracter. That is, there is reason to think that in attentional capture paradigms the exogenous bias is qualitatively identical to that in the model (Fig. 2b, c, f, g) but weaker, so its overt manifestation (in the RT distribution) is more subtle. 
A key consideration is that, during active fixation, as in most studies of covert attention, motor plans are strongly suppressed, so establishing a quantitative link between visual transients, motor activity, and probability of success in a task is more difficult than under urgent conditions. When motor plans are already underway, exogenous capture becomes strong and highly reliable, and its impact on performance easier to discern across tasks (Salinas et al., 2019; Oor et al., 2021; Salinas and Stanford, 2021).

\section{Antisaccade performance requires inhibitory control, but of what?}

Success in the antisaccade task is typically conceptualized as the result of inhibitory control. That is, it is assumed that an inhibitory process suppresses the reflexive reaction of looking at the suddenly-appearing visual cue (Munoz and Everling, 2004; Wiecki and Frank, 2013; Coe and Munoz, 2017). However, our results imply that the precise role played by inhibition in the underlying visuomotor dynamics is by no means obvious.

Under urgent conditions, when fixation is not imposed, our data indicate that the exogenous response to the cue onset is always the same - a transient acceleration of the cue-aligned motor plan. As far as we can tell, there is no mechanism for preventing the corresponding burst of oculomotor activity from occurring; if anything, it is just slightly attenuated in the anti condition relative to the pro (Fig. 4), consistent with differences in the responses of oculomotor neurons across those conditions (Everling et al., 1998; Evering and Munoz, 2000). For the most part, the difference between a successful and an unsuccessful anti trial is simply the degree of development of the ongoing motor plan that is reinforced by the exogenous burst (Fig. 2), which is again consistent with neurophysiology (Everling et al., 1998, 1999; Evering and Munoz, 2000). Although the CAS model does summon inhibition in order to decelerate the incorrect motor plan, such inhibition is a standard constituent of the endogenous signal that informs urgent saccadic choices in general (Stanford et al., 2010; Shankar et al., 2011; Costello et al., 2013; Salinas and Stanford, 2014, 2018, 2021; Seideman et al., 2018; Stanford and Salinas, 2021). Perhaps it partly corresponds to the widespread construct referred to as "cognitive control" (Aron, 2007; Wiecki and Frank, 2013; Cohen, 2017) as it applies to oculomotor behavior. In any case, what is notable is that its action is subsequent to the exogenous response and does not preclude it (Gu et al., 2016; Salinas et al., 2018, 2019; Bompas et al., 2020; Salinas and Stanford, 2021; Stanford and Salinas, 2021).

Under non-urgent conditions, when the cue is presented while fixation is maintained (as in Fig. S1a), antisaccade errors are rare. Successful trials denote a sufficiently strong and sustained fixation signal that resists the exogenous response; and conversely, for an error to be produced, the fixation signal must be disengaged prematurely and/or the exogenous response must be strong enough to overcome it (Everling et al., 1998, 1999; Evering and Munoz, 2000). Either way, it is difficult to determine whether there is a vital target for inhibition that is not just part of the active fixation mechanism.

In conclusion, then, inhibition is critical to antisaccade performance to the extent that it is critical to oculomotor function in general (e.g., for resolving motor conflict; Aron, 2007), but its main point of action during antisaccades, if there is one to be identified, is unclear because it is not the exogenous response itself. This conclusion is consistent with the recent results of Poth (2021), who demonstrated that robust capture phenomena can be generated in urgent tasks that impose very different cognitive demands on the choice process, including conditions in which the competing alternatives are distinguished by visual features alone and not by spatial location. Thus, during urgent perceptual judgments, capture phenomena reveal the temporal evolution of a conflict, not between excitation and inhibition, but between exogenous and endogenous signals that most likely incorporate both. 


\section{Methods}

In general, methods were the same as in the preceding study (Salinas et al., 2019). Here, we summarize key elements of the approach and detail new procedures that were implemented specifically for this experiment.

\section{Subjects and setup}

Experimental subjects were 10 healthy human volunteers, 4 male and 6 female, with a median age of 28 and ranging between 22 and 63 years. They were recruited from the Wake Forest School of Medicine and Wake Forest University communities. All had normal or corrected-to-normal vision. All participants provided informed written consent before the experiment. All experimental procedures were conducted with the approval of the Institutional Review Board (IRB) of Wake Forest School of Medicine.

As in the preceding study (Salinas et al., 2019), the experiment took place in a semi-dark room. The participants sat on an adjustable chair, with their chin and forehead supported, and viewed stimuli presented on a VIEWPixx LED monitor (VPixx Technologies Inc., Saint Bruno, Quebec, Canada; $1920 \times 1200$ screen resolution, $120 \mathrm{~Hz}$ refresh rate, 12-bit color) at a distance of $57 \mathrm{~cm}$. Eye position was recorded using an EyeLink 1000 infrared camera and tracking system (SR Research, Ottawa, Canada; $1000 \mathrm{~Hz}$ sampling rate). For this experiment, stimulus presentation and data collection were controlled using custom Matlab scripts and the Psychtoolbox 3.0 package (Brainard, 1997; Kleiner, 2007).

\section{Behavioral tasks}

The sequence of events was the same in the pro- and antisaccade tasks, and is described in Fig. 1. As in the earlier study (Salinas et al., 2019), the gap values used were $-200,-100,0,75,100$, $125,150,175,200,250$, and $350 \mathrm{~ms}$, where negative numbers correspond to delays in non-urgent versions of the task (Fig. S1); that is, compelled and easy, non-urgent trials were interleaved. In each trial, the gap value and cue location $\left(-8^{\circ}\right.$ or $\left.8^{\circ}\right)$ were randomly sampled. The cue was a circle of $1.5^{\circ}$ diameter. Auditory feedback (a beep) was provided at the end of the trial if the saccadic choice was made within the allowed RT window (425 ms); no sound was played if the limit was exceeded. No feedback was given about the correctness of the spatial choice. The intertrial interval was $350 \mathrm{~ms}$.

Data were collected in blocks of 150 trials, with 2-3 minutes of rest allowed between blocks. For the main experiment, each participant completed 9 or 10 blocks of prosaccade trials, 9 or 10 blocks of antisaccade trials, and 18 blocks of pro and anti trials interleaved. For half of the participants (P1-P5), the cue was green (RGB vector [0 0.88 0]) in prosaccade trials and pink (RGB

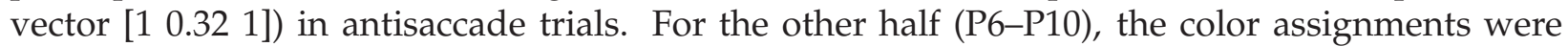
swapped. The green and pink stimuli had the same luminance $\left(48 \mathrm{~cd} \mathrm{~m}^{-2}\right)$, as determined by a spectrophotometer (i1 Pro 2 from X-Rite, Inc., Grand Rapids, MI). In blocks in which pro and anti trials were interleaved, the color of the fixation point was the same as that of the cue, which indicated to the participant the type of trial that was coming up. In blocks of single-task trials, the fixation point was gray (RGB vector [0.25 0.250 .25$]$ ).

\section{Secondary experiment}

Additional data were collected in a secondary experiment in which luminance varied across trials (Fig. 4e). One of the previous volunteers and two newly recruited ones participated (all female). The sequence of events in the tasks were exactly the same as in the main experiment. In this case, however, in each trial, the task type (pro or anti) and cue luminance (high or low) were 
independently sampled, so the 4 possible combinations were randomly interleaved. The color of the fixation spot, which was always the same as that of the upcoming cue, indicated the correct response: green stimuli corresponded to prosaccades and pink stimuli to antisaccades. The green and pink cues of high luminance $\left(48 \mathrm{~cd} \mathrm{~m}^{-2}\right)$ were the same as in the main experiment. The green (RGB vector [0 0.0733 0]) and pink (RGB vector [0.0833 0.0267 0]) cues of low luminance $(0.24 \mathrm{~cd}$ $\mathrm{m}^{-2}$ ) were chosen so that significant rightward shifts of the tachometric curve would be generated (Salinas et al., 2019).

\section{Data analysis}

All results are based on the analysis of urgent trials (gap $\geq 0$ ) only; easy, non-urgent trials (with gap $<0$ ) were excluded. All data analyses were carried out using customized scripts written in Matlab (The MathWorks, Natick, MA), as detailed previously (Salinas et al., 2019).

Processing times were computed as $\mathrm{rPT}=\mathrm{RT}-$ gap, where all quantities are specific to each trial. To generate tachometric curves, trials were grouped into rPT bins that were $21 \mathrm{~ms}$ wide and shifted every $1 \mathrm{~ms}$, and the fraction of correct responses was calculated for the trials contributing to each bin.

To quantify perceptual performance, each tachometric curve was fitted with a continuous analytical function. For the prosaccade task, the fitting curve was an increasing sigmoid

$$
s(x)=B+\frac{A-B}{1+\exp \left(-\frac{x-C}{D}\right)}
$$

where $C$ is the curve rise point, i.e., the rPT at which the fraction correct is halfway between chance (equal to $B$ ) and asymptotic (equal to $A$ ). For the antisaccade task, the fitting curve was defined as

$$
v(x)=\max \left(s_{L}(x), s_{R}(x), 0\right)
$$

where the maximum function, $\max (a, b, c)$, returns the largest of $a, b$, or $c$. Also, $s_{L}$ and $s_{R}$ are two sigmoidal curves given by

$$
\begin{aligned}
& s_{L}(x)=B_{L R}+\frac{A_{L}-B_{L R}}{1+\exp \left(\frac{x-C_{L}}{D_{L}}\right)} \\
& s_{R}(x)=B_{L R}+\frac{A_{R}-B_{L R}}{1+\exp \left(-\frac{x-C_{R}}{D_{R}}\right)}
\end{aligned}
$$

where $s_{L}$ tracks the left (decreasing) side of the tachometric curve and $s_{R}$ tracks the right (increasing) side. In this case, $C_{L}$ is the curve drop point, i.e., the $\mathrm{rPT}$ at which the fraction correct is halfway between chance (equal to $A_{L}$ ) and minimum (equal to $B_{L R}$ ), whereas $C_{R}$ is the curve rise point, which in this case corresponds to the rPT at which the fraction correct is halfway between the minimum $\left(B_{L R}\right)$ and asymptotic $\left(A_{R}\right)$. Previously (Salinas et al., 2019), we referred to the antisaccade rise point as the curve centerpoint, but the new nomenclature is more clear and more suitable for comparing results between pro and anti conditions.

For any given tachometric curve, parameter values $(A, B, C$, etc.) were found that minimized the mean absolute error between the experimental data and the analytical curves using the Matlab function fminsearch. In most cases, chance levels were fixed at $50 \%$ correct by setting $B=$ 0.5 and $A_{L}=0.5$, so these parameters did not vary during the minimization. However, when analyzing motor biases (Fig. 5), these parameters were allowed to vary.

In this study, we focus on the drop point $C_{L}$ and the rise points $C$ and $C_{R}$. Confidence intervals for these quantities were obtained by bootstrapping (Davison and Hinkley, 2006; Hesterberg, 
2014), as done previously (Salinas et al., 2019). This involved resampling the trial-wise data with replacement, refitting the resulting (resampled) tachometric curves, storing the new parameter values, and repeating the process many times to generate parameter distributions. Reported 95\% confidence intervals correspond to the 2.5 and 97.5 percentiles obtained from the bootstrapped distributions based on 1,000 - 10,000 iterations.

In addition to comparisons between $C, C_{L}$, and $C_{R}$ values, to evaluate differences in timing between two tachometric curves (as in Fig. $4 \mathrm{~b}-\mathrm{d}$ ), we also performed a time-shift analysis, which was as follows. First, one curve, $f_{1}(x)$, was designated as the reference and remained fixed. Then, transformed versions of the second curve, $f_{2}(x)$, were considered that would minimize the absolute difference between it and the reference curve over a range of $x$ values (that is, rPTs). Three transforming operations were considered: a shift along the y axis (i.e., a change in baseline), a change in gain, and a shift along the $\mathrm{x}$ axis. In practice, values of the parameters $\Delta b, g$, and $\Delta x$ were found that minimized the error

$$
E=\left\langle\left|g\left(\Delta b+f_{2}(x+\Delta x)\right)-f_{1}(x)\right|\right\rangle
$$

where the angle brackets indicate an average over all rPT values in the specified range. For identical tachometric curves $f_{1}$ and $f_{2}$ the optimal parameters would be $\Delta b=0, g=1$, and $\Delta x=0$. Although the quantity of interest is the relative time shift between the curves, $\Delta x$, the gain factor and change in baseline are included to account for the fact that the two curves may saturate at somewhat different levels, or may be slightly offset in the vertical direction from each other, which would tend to overestimate the magnitude of the optimal shift, i.e., the $\Delta x$ for which the error is minimized. Notably, the effect of $\Delta b$ and $g$ was modest; results varied by approximately 2 $\mathrm{ms}$ when the procedure allowed variations in $\Delta x$ only, in which case $\Delta b$ and $g$ were fixed at 0 and 1 , respectively.

This shift analysis applies to curves $f_{1}$ and $f_{2}$ that have similar shapes. To compare the early departure from chance in pro- versus antisaccade curves, the prosaccade curve was first inverted (Fig. $4 \mathrm{~b}, \mathrm{c}$ ) relative to the chance level by applying the transformation $f(x) \rightarrow 1-f(x)$. Then the above procedure for determining $\Delta x$ was carried out. Confidence intervals for $\Delta x$ were again obtained by bootstrapping, i.e., by resampling the data with replacement, recalculating the tachometric curves $f_{1}$ and $f_{2}$, and rerunning the entire minimization process. In this way, by iterating this resampling procedure many times, distributions of $\Delta x$ values were generated.

\section{Model simulations}

Predictions were made with the CAS and CPS models as explained in the Results. The CAS model is the exact same accelerated race-to-threshold model developed previously (for parameter values, see Table 1 in Salinas et al., 2019, high-luminance cue condition). The CAS model thus represents typical, or average, urgent antisaccade performance, as it replicates the psychometric results obtained by pooling all the trials from the 6 subjects that participated in the prior experiment. The Matlab scripts for simulating the CAS and CPS models differed by a single sign that determined whether the target location was equal to the cue location or opposite to it. Thus, for a given set of parameters, the exogenous and endogenous modulations produced by the two models were the same; the only thing that varied was which plan was accelerated and which decelerated endogenously. To generate predictions, the CPS model was run with the same parameter values as the CAS model.

In standard model simulations with no motor bias, the two initial buildup rates for the competing motor plans were drawn from a bivariate distribution and were assigned randomly to the cue and anti motor plans. Model runs in which a motor bias was included proceeded in the same way, 
with initial buildup rates drawn from the same bivariate distribution, but these were assigned to the cue and the anti plans with a biased probability. For example, to generate a bias of 0.1 in favor of the cue location, in each simulated trial the larger of the two buildup rates was assigned to the cue side with a $60 \%$ probability. Biases of arbitrary magnitude favoring one direction or the other were generated this way.

\section{References}

Aron AR (2007) The neural basis of inhibition in cognitive control. Neuroscientist 13:214-228.

Bompas A, Campbell AE, Sumner P (2020) Cognitive control and automatic interference in mind and brain: A unified model of saccadic inhibition and countermanding. Psychol Rev 127:524561.

Brainard DH (1997). The psychophysics toolbox. Spatial Vision 10:433-436.

Busse L, Katzner S, Treue S (2008) Temporal dynamics of neuronal modulation during exogenous and endogenous shifts of visual attention in macaque area MT. Proc Natl Acad Sci USA 105:16380-16385.

Coe BC, Munoz DP (2017) Mechanisms of saccade suppression revealed in the anti-saccade task. Philos Trans R Soc Lond B Biol Sci 372(1718), pii: 20160192.

Cohen JD (2017) Cognitive control: core constructs and current considerations. In: Egner T, editor. The Wiley Handbook of Cognitive Control. Chichester, UK: John Wiley \& Sons, Ltd. pp. $1-28$.

Costello MG, Zhu D, Salinas E, Stanford TR (2013) Perceptual modulation of motor - but not visual - responses in the frontal eye field during an urgent-decision task. J Neurosci 33:16394-16408.

Everling S, Dorris MC, Klein RM, Munoz DP (1999) Role of primate superior colliculus in preparation and execution of anti-saccades and pro-saccades J Neurosci 19:2740-2754.

Everling S, Dorris MC, Munoz DP (1998) Reflex suppression in the anti-saccade task is dependent on prestimulus neural processes. J Neurophysiol 80:1584-1589.

Everling S, Munoz DP (2000) Neuronal correlates for preparatory set associated with pro-saccades and anti-saccades in the primate frontal eye field. J Neurosci 20:387-400.

Gu C, Wood DK, Gribble PL, Corneil BD (2016) A trial-by-trial window into sensorimotor transformations in the human motor periphery. J Neurosci 36:8273-8282.

Herrington TM, Assad JA (2010) Temporal sequence of attentional modulation in the lateral intraparietal area and middle temporal area during rapid covert shifts of attention. J Neurosci 30:3287-3296.

Itti L, Koch C (2001) Computational modelling of visual attention Nat Rev Neurosci 2:194-203.

Kalesnykas RP, Hallett PE (1987) The differentiation of visually guided and anticipatory saccades in gap and overlap paradigms. Exp Brain Res 68:115-121.

Kleiner M, Brainard D, Pelli D (2007) Whats new in Psychtoolbox-3? Perception 36 (ECVP Abstract Supplement).

Monsell S (2003) Task switching. Trends Cogn Sci 7:134-140.

Munoz DP, Everling S (2004) Look away: the anti-saccade task and the voluntary control of eye 
movement. Nat Rev Neurosci 5:218-228.

Oor EE, Salinas E, Stanford TR (2021) Encoding of salience and informed choice colludes and conflicts across FEF neuronal types in the guidance of urgent saccadic decisions. Soc Neurosci Abstr, Prog No 491.05.

Paré M, Munoz DP (1996) Saccadic reaction time in the monkey: advanced preparation of oculomotor programs is primarily responsible for express saccade occurrence. J Neurophysiol 76:3666-3681.

Poth CH (2021) Urgency forces stimulus-driven action by overcoming cognitive control. Elife 10:e73682.

Ruz M, Lupiáñez J (2002) A review of attentional capture: On its automaticity and sensitivity to endogenous control. Psicológica 23:283-309.

Salinas E, Stanford TR (2018) Saccadic inhibition interrupts ongoing oculomotor activity to enable the rapid deployment of alternate movement plans. Sci Rep 8:14163.

Salinas E, Stanford TR (2021) Under time pressure, the exogenous modulation of saccade plans is ubiquitous, intricate, and lawful. Curr Opin Neurobiol 70:154-162.

Salinas E, Steinberg BR, Sussman LA, Fry SM, Hauser CK, Anderson DD, Stanford TR (2019) Voluntary and involuntary contributions to perceptually guided saccadic choices resolved with millisecond precision. eLife 8:e46359.

Seideman JA, Stanford TR, Salinas E (2018) Saccade metrics reflect decision-making dynamics during urgent choices. Nat Commun 9:2907.

Shankar S, Massoglia DP, Zhu D, Costello MG, Stanford TR, Salinas E (2011) Tracking the temporal evolution of a perceptual judgment using a compelled-response task. J Neurosci 31:84068421.

Sparks D, Rohrer WH, Zhang Y (2000) The role of the superior colliculus in saccade initiation: a study of express saccades and the gap effect. Vision Res 40:2763-2777.

Stanford TR, Salinas E (2021) Urgent decision making: resolving visuomotor interactions at high temporal resolution. Annu Rev Vis Sci 7:323-348.

Stanford TR, Shankar S, Massoglia DP, Costello MG, Salinas E (2010) Perceptual decision making in less than 30 milliseconds. Nat Neurosci 13:379-385.

Theeuwes J (2010) Top-down and bottom-up control of visual selection. Acta Psychol (Amst) 135:77-99.

Theeuwes J, Kramer AF, Hahn S, Irwin DE (1998) Our eyes do not always go where we want them to go: capture of the eyes by new objects. Psychol Sci 9:379-385.

Theeuwes J, Kramer AF, Hahn S, Irwin DE, Zelinsky GJ (1999) Influence of attentional capture on oculomotor control. J Exp Psychol Hum Percept Perform 25:1595-1608.

Wiecki TV, Frank MJ (2013) A computational model of inhibitory control in frontal cortex and basal ganglia. Psychol Rev 120:329355.

Wolfe JM, Horowitz TS (2017) Five factors that guide attention in visual search. Nature Hum Behav 1:0058.

Wylie G, Allport A (2000) Task switching and the measurement of "switch costs". Psychol Res 63:212-233. 

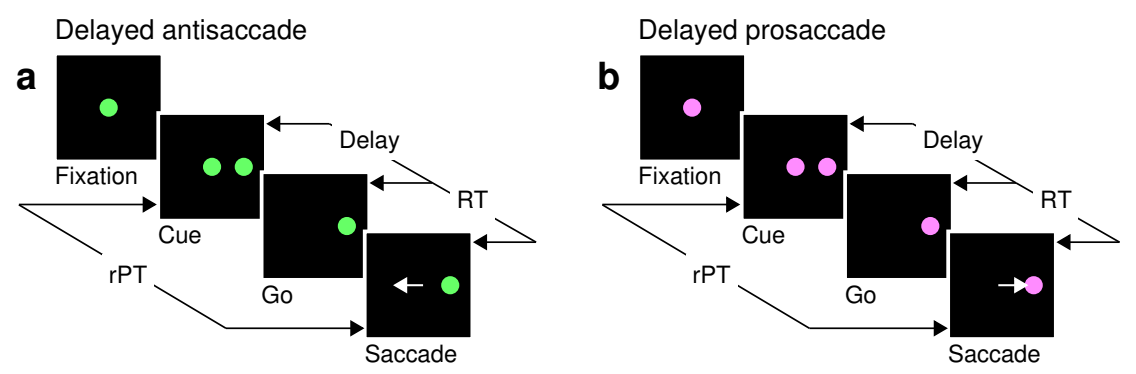

Figure S1. Non-urgent variants of the tasks. a, The delayed antisaccade task. After a fixation period $\left(500,600\right.$, or $700 \mathrm{~ms}$ ), the cue is revealed (Cue, $\pm 8^{\circ}$ ). After a delay period (Delay, 100 or $200 \mathrm{~ms}$ ), the central fixation point disappears (Go), instructing the participant to make an eye movement within $425 \mathrm{~ms}$. The correct response is to look away from the cue, to the diametrically opposite location (Saccade, white arrow). b, The delayed prosaccade task. The sequence of events is the same as for the delayed antisaccade task, except that the correct response is an eye movement toward the cue. In all trials, the cue location and delay are selected randomly; the reaction time (RT) is measured between the onset of the go signal and the onset of the saccade; and the raw processing time $(\mathrm{rPT})$ is measured between cue onset and saccade onset (calculated as Delay + $R T)$. 

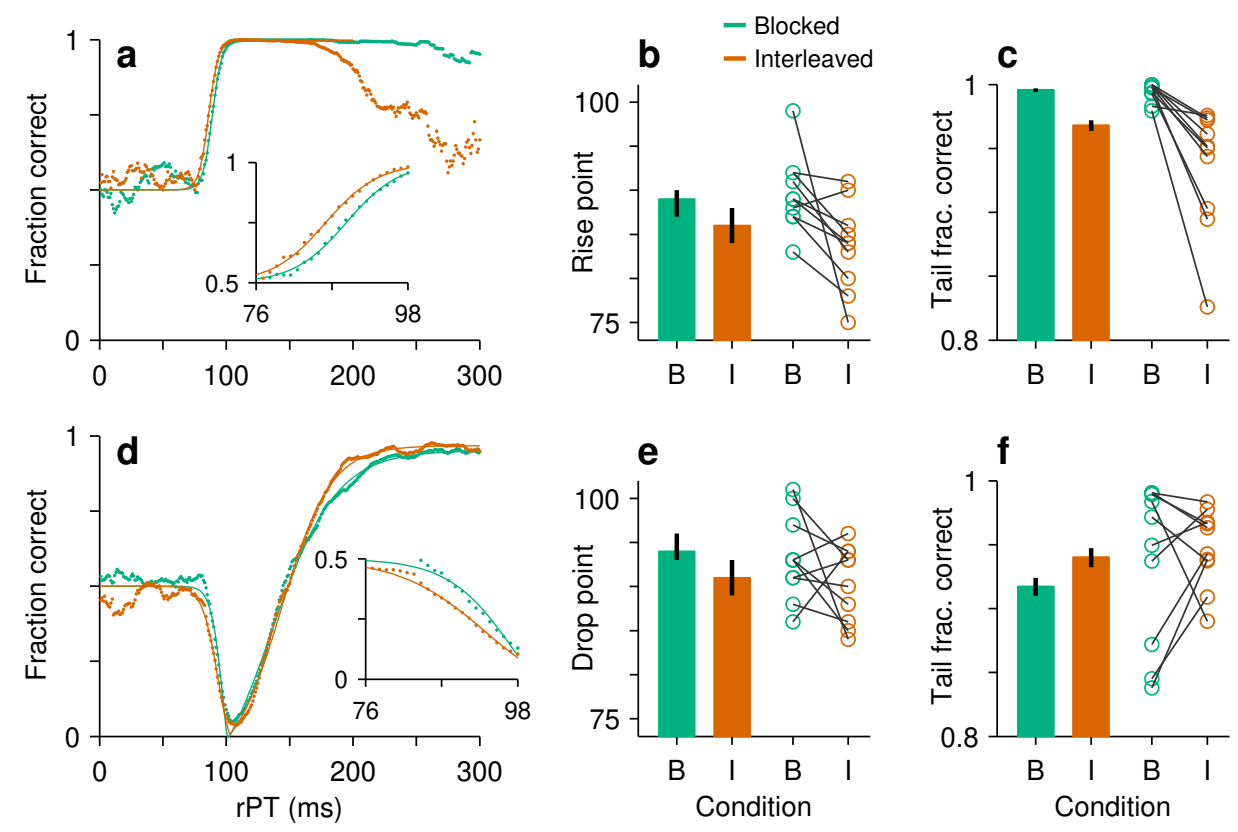

Figure S2. Performance in blocked versus interleaved trials. In all panels, colors mark data collected in blocks of same-task trials (green) or in blocks in which pro and anti trials were randomly interleaved (orange). a, Tachometric curves for prosaccade trials pooled across participants. Data points show the fraction of correct choices for each $\mathrm{rPT}$ bin (bin width $=21 \mathrm{~ms}$ ). Continuous lines are sigmoidal fits. The inset zooms into the rPT range over which performance departs sharply from chance. $\mathbf{b}$, Curve rise point, which is the rPT at which prosaccade performance is halfway between chance and asymptotic. Bars show values for the pooled tachometric curves (in a), with 95\% CIs (error bars, from bootstrap). Circles are individual participant data ( $p=0.006$ for difference between conditions, signed rank test). c, Fraction correct at the tail of the rPT range (rPT $\geq$ $125 \mathrm{~ms}$ ). Same format as in $\mathbf{b}$. For the individual participant data, $p=0.002$ (signed rank test). $\mathbf{d}$, As in $\mathbf{a}$, but for antisaccade trials. e, As in $\mathbf{b}$, but for the drop point, which is the rPT at which antisaccade performance is halfway between chance and its minimum value. For the individual participant data, $p=0.2$ (signed rank test). f, As in c, but for antisaccade trials (rPT $\geq 190 \mathrm{~ms}$ ). For the individual participant data, $p=0.6$ (signed rank test). 

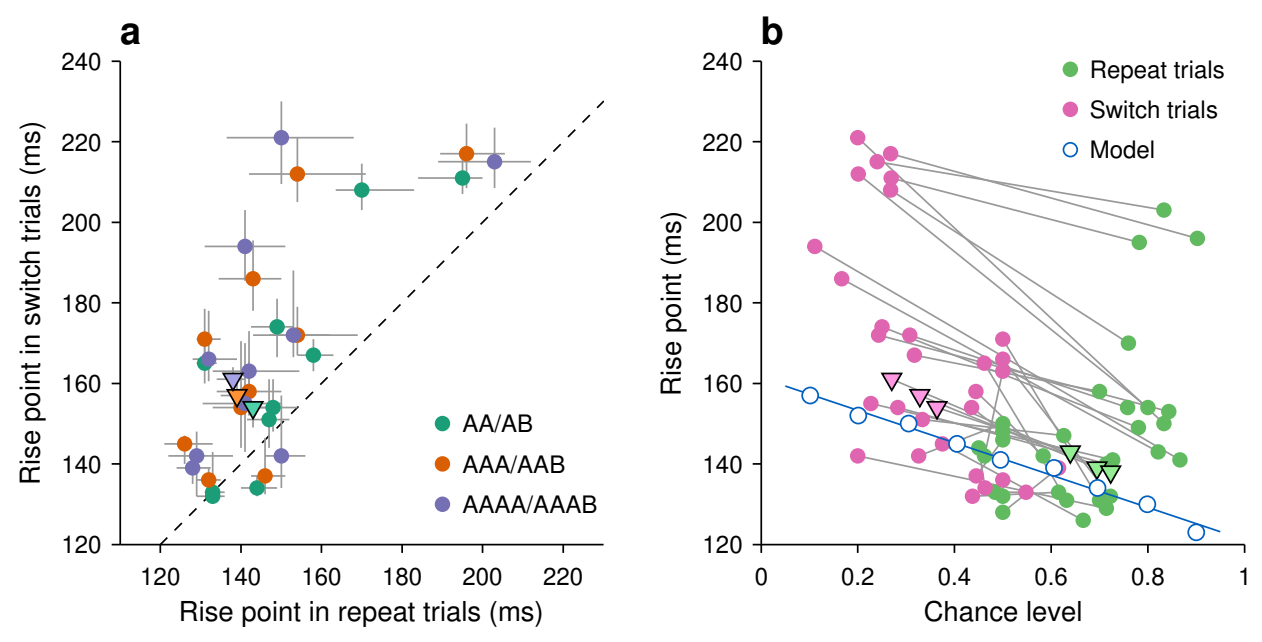

Figure S3. Effect of motor bias on antisaccade performance in individual participants. a, Rise point of the tachometric curve for trials in which the target switched locations (y axis) versus trials in which the target location was repeated (x axis). Colors indicate results for trial histories going back 1 (green), 2 (orange), or 3 trials (purple). Data points are for individual participants (circles) and for trials pooled across participants (triangles; from the curves in Fig. 5a-c). Gray lines indicate 95\% CIs from bootstrap. b, Rise point of the tachometric curve as a function of the chance level of the same curve, which corresponds to the probability correct for guesses (rPT $\lesssim 80 \mathrm{~ms}$ ). Data points are for individual participants (filled circles), for trials pooled across participants (triangles; from the curves in Fig. 5a-c), and from simulations of the CAS model (open blue circles; blue line is linear fit to the simulated data points). Each participant contributed 3 points from repeat trials (pink) and 3 points from switch trials (green). Gray lines join data from the same participant in a given condition (history going back 1,2, or 3 trials). Note that, in spite of the visible individual variability, there is a clear trend across participants for a negative correlation between rise point and chance level that is highly consistent with that predicted by the model. 